\title{
A Diffusion and Reaction Related Model of the Epitaxial Lift-Off Process
}

\author{
A. T. J. van Niftrik, ${ }^{\text {a, }}$ J. J. Schermer, ${ }^{\text {a }}$ G. J. Bauhuis, ${ }^{a}$ P. Mulder, ${ }^{a}$ P. K. Larsen, ${ }^{a}$ \\ and J. J. Kelly ${ }^{\mathrm{b}, *}$ \\ ${ }^{a}$ Applied Materials Science, Institute for Molecules and Materials, Radboud University Nijmegen, \\ 6525 ED Nijmegen, The Netherlands \\ ${ }^{b}$ Condensed Matter and Interfaces, Debye Institute, Utrecht University, 3508 TA Utrecht, The Netherlands
}

\begin{abstract}
In the present work a so-called diffusion and reaction related model (DR model) is derived based on the notion that the overall etch rate in the epitaxial lift-off (ELO) process is determined both by the diffusion rate of hydrofluoric acid to the etch front and its subsequent reaction rate in the process. In contrast to the model that was previously described in the literature, the DR model yields etch rates which are in quantitative agreement with those obtained experimentally. In order to verify the DR model, the ELO etch rate of $\mathrm{AlAs}_{1-y} \mathrm{P}_{y}$ release layers is determined as a function of the phosphorus percentage, the release layer thickness and the temperature. In accordance with the DR model, it is shown that the etch rate is reaction rate related by the dependence on the phosphorus percentage in the release layer, and that the etch rate is diffusion rate related by the dependence on the release layer thickness. From the temperature dependence, an activation energy of $0.31 \mathrm{eV}$ could be deduced for the ELO process under the present conditions.
\end{abstract}

(c) 2007 The Electrochemical Society. [DOI: 10.1149/1.2779968] All rights reserved.

Manuscript submitted March 23, 2007; revised manuscript received July 26, 2007. Available electronically September 19, 2007.

The "epitaxial lift-off" (ELO) technique (see Fig. 1a), in which a III/V device structure is separated from its GaAs substrate by using selective wet etching of a thin $\mathrm{Al}_{x} \mathrm{Ga}_{1-x} \mathrm{As}(x>0.6)$ release layer and transferred to a foreign carrier, allows the production of singlecrystalline thin films of III/V materials. ${ }^{1}$ Application of this technique is interesting for the optoelectronics industry, because use of thin-film devices potentially results in a more efficient transfer of generated heat from device to carrier or heat sink and significantly reduces the amount of material needed by reuse of the substrates. ${ }^{2}$ This is of particular importance for an intrinsically large area, thus expensive devices like high efficiency III/V solar cells, ${ }^{2,3}$ and the integration of III/V based components with, e.g., silicon-based devices. ${ }^{4,5}$ Recently, at our institute thin-film GaAs solar cells were made based on the ELO technique, which reached record efficiencies of $24.5 \%$. ${ }^{6}$ This is close to the highest efficiency of $25.1 \%$ reported for regular GaAs cells on a GaAs substrate, ${ }^{7}$ which indicates that the ELO process is not detrimental to the quality of the thin-film device.

In 1978 Konagai et al. ${ }^{8}$ described the separation of devices from a GaAs substrate using the extreme selectivity of hydrofluoric acid (HF) for $\mathrm{Al}_{x} \mathrm{Ga}_{1-x} \mathrm{As}$ with a high $\mathrm{Al}$ fraction. A wax layer was applied to support the $30 \mu \mathrm{m}$ thick fragile films during the process. Yablonovitch et al. $^{9}$ noted that the tension induced by the wax caused the thinner films, of micrometer thickness, to curl up with a radius of curvature $r$ as they became undercut. This was concluded to be beneficial for removal of the etch products, leading to an increased lateral etch rate $V_{\mathrm{e}}$ of the AlAs release layer. By assuming that three moles of hydrogen $\left(\mathrm{H}_{2}\right)$ gas are produced for each mole of AlAs etched and that the ability of dissolved $\mathrm{H}_{2}$, which has a low solubility, to diffuse away limits the etch rate during the ELO process, they derived an expression for $V_{\mathrm{e}}$

$$
V_{e}=\frac{\sqrt{2}}{\pi \sqrt{r h}} \frac{D\left[\mathrm{H}_{2}\right]}{3[\mathrm{AlAs}]}
$$

where $\left[\mathrm{H}_{2}\right]$ and $[\mathrm{AlAs}]$ are the molar concentrations of dissolved $\mathrm{H}_{2}$ and solid AlAs, $D$ the diffusion coefficient of $\mathrm{H}_{2}$ and $h$ the release layer thickness. Based on Eq. 2, Maeda et al. ${ }^{4}$ argued that $V_{\mathrm{e}}$ in Eq. 1 increases exponentially with temperature $T$ as a result of the temperature dependence of $D$

\footnotetext{
Electrochemical Society Active Member

${ }^{\mathrm{z}}$ E-mail: ton.vanniftrik@science.ru.nl
}

$$
D=D_{0} \exp \left(\frac{-E_{\mathrm{a}, \mathrm{d}}}{k_{\mathrm{B}} T}\right)
$$

where $D_{0}$ is the diffusion coefficient at infinite temperature, $E_{\mathrm{a}, \mathrm{d}}$ the activation energy, associated with the diffusion of $\mathrm{H}_{2}$, and $k_{\mathrm{B}}$ the Boltzmann constant.

According to the Yablonovitch model, the maximum etch rate is reached when the concentration of dissolved $\mathrm{H}_{2}$ at the etch front equals the maximum allowable concentration of $\mathrm{H}_{2}$ (i.e., the solubility of $\left.\mathrm{H}_{2}\right)$ in the solution $\left(\left[\mathrm{H}_{2}\right]_{\max }=0.78 \times 10^{-3} \mathrm{M}\right.$ at $\left.298 \mathrm{~K}^{10}\right)$. For the diffusion of $\mathrm{H}_{2}, D$ is given by $5.11 \times 10^{-5} \mathrm{~cm}^{2} / \mathrm{s}$ at $298 \mathrm{~K}$ and $E_{\mathrm{a}, \mathrm{d}}$ is calculated to be $0.167 \mathrm{eV} .^{10}$ The molar concentration of AlAs $N$ is given by $1 /($ molar volume of AlAs $)=($ density of AlAs $) /$ (molar mass of AlAs). With a density of $3.729 \mathrm{~g} / \mathrm{cm}^{3}$ and a molar mass of $101.9 \mathrm{~g} / \mathrm{mol},{ }^{10} \mathrm{~N}$ is calculated to be $36.6 \mathrm{M}$. In the past, the ELO process was typically conducted with $r=50 \mathrm{~mm}, h=5 \mathrm{~nm}$ and $T=298 \mathrm{~K}$. Under these conditions an etch rate of $3.7 \mu \mathrm{m} / \mathrm{h}$ is

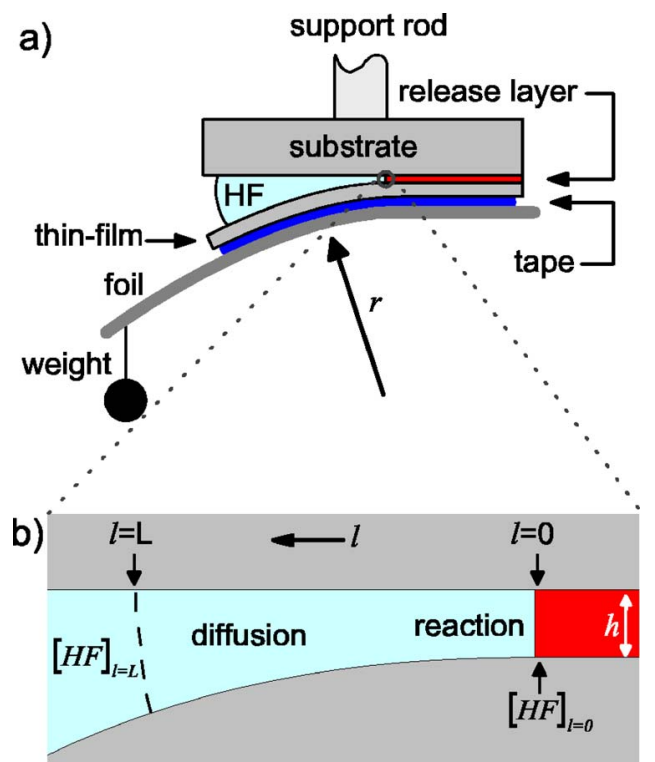

Figure 1. (Color online) Schematic cross section of the (a) weight induced ELO (WIELO) setup, and (b) detail of figure (a) indicating the diffusion and reaction regime of the process. Note that the dimensions are not to scale. 
predicted by the model. In the actual experiments, however, much larger etch rates of several $\mathrm{mm} / \mathrm{h}$ were obtained. ${ }^{11-13}$

Nevertheless, the experiments ${ }^{11}$ did show qualitatively the predicted linear relation between $V_{\mathrm{e}}$ and $h^{-0.5}$, indicating that at least under some conditions diffusion does play a limiting role in the etch process. On the other hand, there is also experimental evidence that points to a reaction rate related process. It was, for example, found that the etch rate could be varied significantly by the introduction of strain to the system. The strain could either be applied using an external force (bending the sample during etching ${ }^{14}$ ) or an intrinsic force (application of strained layers surrounding the AlAs release layers $\left.{ }^{13}\right)$. In the present work a model is derived based on the idea that the overall etch rate in the ELO process is determined both by the diffusion rate and the subsequent reaction rate of a certain species in the process. Furthermore, this diffusion and reaction related model (DR model) is tested with several series of experiments.

Recently, it was shown that arsine $\left(\mathrm{AsH}_{3}\right)$ instead of hydrogen is produced during the ELO process. ${ }^{15}$ It was found that the reaction between AlAs and the HF etchant is most likely described by a set of overall reactions given by

$$
\begin{aligned}
\mathrm{AlAs}+3 \mathrm{HF}+6 \mathrm{H}_{2} \mathrm{O} \rightarrow & \mathrm{AsH}_{3}+\left[\mathrm{AlF}_{n} \cdot\left(\mathrm{H}_{2} \mathrm{O}\right)_{6-n}\right]^{(3-n)+} \\
& +(3-n) \mathrm{F}^{-}+n \mathrm{H}_{2} \mathrm{O}
\end{aligned}
$$

with $n=0,1,2,3$. So instead of $\mathrm{H}_{2}$, the ELO etch rate should be determined by either $\mathrm{HF}, \mathrm{AsH}_{3}$ or one of the aluminium fluoride complexes. In $\mathrm{HF}$ solutions, gaseous $\mathrm{AsH}_{3}$ is expected to have a low solubility of $\sim 1 \times 10^{-3} \mathrm{M}$, comparable to the solubility in water. ${ }^{16}$ Hence, in a similar way as shown for $\mathrm{H}_{2}$ in the introduction, diffusion of $\mathrm{AsH}_{3}$ would limit the ELO etch rate to a maximum of only a few micrometers per hour under the applied conditions. Because the experimentally obtained etch rates are several orders of magnitude larger, we have to assume that $\mathrm{AsH}_{3}$ is removed quickly from the solution, e.g., via gas bubble transport. In HF solutions, HF and the aluminium fluoride complexes have a high solubility $(>1 \mathrm{M})$. We assume that HF limits the overall etch rate in the ELO process and that the aluminium fluoride complexes are removed quickly from the etch front.

According to Eq. 3, the etch process consumes three moles of HF per mole of etched AlAs. The concentration of HF is the lowest at the etch front where it is consumed $\left([\mathrm{HF}]_{\ell=0}\right)$ and gradually increases with the distance from the etch front until the bulk concentration $\left([\mathrm{HF}]_{\ell=L}\right.$ ) is reached at $\ell=L$ (see Fig. 1b). In this way the AlAs etch rate related to the diffusion of HF becomes

$$
V_{\mathrm{e}, \mathrm{d}}=\frac{\left([\mathrm{HF}]_{\ell=L}-[\mathrm{HF}]_{\ell=0}\right)}{R_{\mathrm{d}}}
$$

with $\left([\mathrm{HF}]_{\ell=L}-[\mathrm{HF}]_{\ell=0}\right)$ as concentration difference and $R_{d}$ the diffusion related resistance. In a similar way as deduced for the diffusion of $\mathrm{H}_{2}$ (see Eq. 1 and 2), it can be shown that

$$
R_{\mathrm{d}}=\frac{\pi \sqrt{r h} 3[\mathrm{AlAs}]}{\sqrt{2} D_{0}} \exp \left(\frac{E_{\mathrm{a}, \mathrm{d}}}{k_{B} T}\right)
$$

where [AlAs] is the molar concentrations of AlAs, $h$ the release layer thickness, $r$ the radius of curvature of the thin film, $D_{0}$ is the diffusion coefficient at infinite temperature and $E_{\mathrm{a}, \mathrm{d}}$ the activation energy associated with the diffusion of HF.

During the last few years, experiments were performed without diffusion limitations. ${ }^{12,14}$ These experiments, the so-called bulk-etch experiments, showed that in the absence of restrictions imposed by the diffusion process the reaction-limited etch rate of $\mathrm{Al}_{x} \mathrm{Ga}_{1-x} \mathrm{As}$ layers depends on the $\mathrm{Al}$ fraction, the $\mathrm{HF}$ concentration, and the strain on the layers. For $\mathrm{Al}_{x} \mathrm{Ga}_{1-x}$ As layers over a composition range from $x=0.65$ to $x=1$, it was shown that the etch rate increases linearly with the HF concentration. ${ }^{12}$ Based on this result, the reaction related etch rate $V_{\mathrm{e}, \mathrm{r}}$ for $\mathrm{AlAs}$ can be expressed as

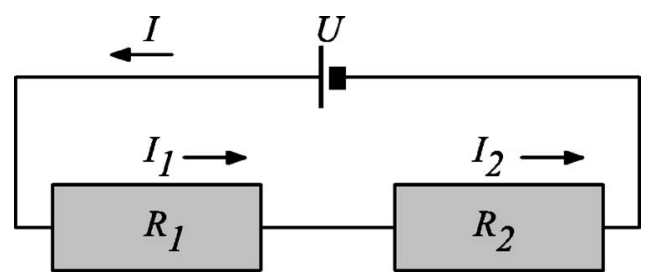

Figure 2. An electric circuit with two resistances, $R_{1}$ and $R_{2}$, coupled in series. Note, that the current through both resistances is the same, and equal to $I=U /\left(R_{1}+R_{2}\right)$, with $U$ the potential

$$
V_{\mathrm{e}, \mathrm{r}}=-\frac{d \mathrm{AlAs}}{d t}=k[\mathrm{HF}]_{\ell=0}
$$

where $[\mathrm{HF}]_{\ell=0}$ is the concentration of HF at the etch front and $k$ the reaction constant. It should be noted that $k$ increases exponentially with temperature according to

$$
k=A \exp \left(\frac{-E_{\mathrm{a}, \mathrm{r}}}{k_{\mathrm{B}} T}\right)
$$

where $A$ is the Arrhenius constant and $E_{\mathrm{a}, \mathrm{r}}$ the activation energy associated with the reaction barrier. Rewriting of Eq. 6 and 7 results in the following expressions

$$
V_{\mathrm{e}, \mathrm{r}}=\frac{[\mathrm{HF}]_{\ell=0}}{R_{\mathrm{r}}}
$$

with $R_{\mathrm{r}}$ the reaction rate resistance given by

$$
R_{\mathrm{r}}=\frac{1}{A} \exp \left(\frac{E_{\mathrm{a}, \mathrm{r}}}{k_{\mathrm{B}} T}\right)
$$

The overall etch rate for the ELO process is determined by both the diffusion of HF towards the etch front and its reaction with the release layer. Analog to an electric circuit with two resistances, $R_{1}$ and $R_{2}$, coupled in series (see Fig. 2) where the currents through both resistances are the same and equal the overall current, $I=I_{1}$ $=I_{2}$, the overall etch rate $V_{\mathrm{e}}$ is given by

$$
V_{\mathrm{e}}=V_{\mathrm{e}, \mathrm{d}}=V_{\mathrm{e}, \mathrm{r}}
$$

Substitution of Eq. 4 and 8 results in an expression for the concentration of $\mathrm{HF}$ at the etch front $[\mathrm{HF}]_{\ell=0}$

$$
[\mathrm{HF}]_{\ell=0}=\frac{R_{\mathrm{r}}}{R_{\mathrm{d}}+R_{\mathrm{r}}}[\mathrm{HF}]_{\ell=L}
$$

Substitution of Eq. 10 and 11 in the expressions for $V_{\mathrm{e}, \mathrm{d}}$ or $V_{\mathrm{e}, \mathrm{r}}$ yields

$$
V_{\mathrm{e}}=\frac{[\mathrm{HF}]_{\ell=L}}{R_{\mathrm{d}}+R_{\mathrm{r}}}
$$

in which, analog to an electric circuit, $V_{\mathrm{e}}$ is the current $I,[\mathrm{HF}]_{\ell=L}$ the potential $U$ and $R_{\mathrm{d}}+R_{\mathrm{r}}$ the total resistance of the process.

\section{Experimental}

The layer structure of the samples used in this study is schematically represented in Fig. 3. A series of samples with $10 \mathrm{~nm}$ thick release layers and small $\mathrm{P}$ fractions ranging from $y=0.00$ to $y$ $=0.08$ (the $\mathrm{AlAs}_{1-y} \mathrm{P}_{y}$ series) is used to study the influence of misfit strain on the etch process. In the case of a layer coherently grown on top of a relatively thick substrate, the in-plane strain $\varepsilon$ on the $\mathrm{AlAs}_{1-y} \mathrm{P}_{y}$ release layers is described by

$$
\varepsilon=\frac{a_{\mathrm{sub}}-a_{\mathrm{rel}}}{a_{\mathrm{rel}}}
$$

where $a_{\text {sub }}$ and $a_{\text {rel }}$ refer to the unstrained lattice parameters of substrate and release layer, respectively. The strain can be either compressive $\left(a_{\text {sub }}<a_{\text {rel }}\right)$ or tensile $\left(a_{\text {sub }}>a_{\text {rel }}\right)$. Note, that the lattice 


\begin{tabular}{|cl|}
\hline $1 \mu \mathrm{m}$ & GaAs thin-film \\
\hline$h \mathrm{~nm}$ & AlAs $_{1-\mathrm{y}} \mathrm{P}_{\mathrm{y}}$ release layer \\
\hline $160 \mathrm{~nm}$ & GaAs buffer layer \\
\hline & \\
& \\
$350 \mu \mathrm{m}$ & GaAs substrate \\
& \\
& \\
\hline
\end{tabular}

Figure 3. Schematic cross section of the grown layer structures with the thin-film GaAs layer and GaAs substrate (plus buffer layer) separated by an $\mathrm{AlAs}_{1-y} \mathrm{P}_{y}$ layer.

constants of the ternary compositions are determined from those of the binary compounds, taken from Sze ${ }^{17}$ (see Table I), using Vegard's law. A second sample series (the thickness series) with a $\mathrm{P}$ content corresponding to the maximum lateral etch rate $(y=0.02)$ and release layer thicknesses $h$ varying from 3 to $40 \mathrm{~nm}$, is used to study the diffusion related part of the ELO process. Previous experiments ${ }^{11}$ showed that the ELO etch rate $V_{\mathrm{e}}$ linearly depends on $h^{-0.5}$, but the interpretation of these results was partly hampered because the single HF droplet, used to etch the layers, evaporated before the ELO process was completed. Therefore, these experiments were repeated for the present work utilizing an improved ELO setup. This setup allows for a continuous flow of the HF solution towards the sample and has an improved temperature control system. A third sample series (the temperature series) having both the optimum $\mathrm{P}$ composition and the optimum release layer thickness $(10 \mathrm{~nm})$ is used for ELO experiments performed at temperatures ranging from 23.4 to $71.2^{\circ} \mathrm{C}$. For convenience, the samples in this work will be referred to by the composition of the release layer, the release layer thickness or the temperature at which the ELO process was performed.

All samples were grown on 2 in. diam undoped GaAs wafers with crystal orientation (001), $2^{\circ}$ off towards $\langle 110\rangle$ using lowpressure metallorganic chemical vapor deposition in a horizontal Aixtron 200 reactor. Source materials were trimethyl-gallium and trimethyl-aluminium as group-III precursors and arsine and phosphine as group- $\mathrm{V}$ precursors. Disilane was used as dopant precursor to obtain n-type doping for the $\mathrm{AlAs}_{1-y} \mathrm{P}_{y}$ release layers; all other layers were undoped. Each growth run was performed at a temperature of $650^{\circ} \mathrm{C}$ and a pressure of $20 \mathrm{mbar}$. Under these conditions the $\mathrm{AlAs}_{1-y} \mathrm{P}_{y}$ release layer was grown at a rate of $\sim 1.5 \mu \mathrm{m} / \mathrm{h}$ (V/III ratio ranging from 192 to 255 depending on the $\mathrm{P}$ concentration), and the GaAs layers were deposited at $1.87 \mu \mathrm{m} / \mathrm{h}$ (V/III ratio $=121$ ). The solid composition of the $\mathrm{AlAs}_{1-y} \mathrm{P}_{y}$ layers was determined by X-ray diffraction using a Bruker D8 diffractometer.

After deposition, the wafers were cleaved in pieces of 10 $\times 10 \mathrm{~mm}^{2}$. Then, a flexible carrier was mounted on top of the samples using double sided tape. The samples were etched using a weight induced ELO (WIELO) process (see Fig. 1a). In this process

Table I. Lattice constants (at $300 \mathrm{~K}$ ) of the binary compounds used in the calculations.

Binary compound Lattice constant $(\AA)$

$\begin{array}{ll}\text { AlAs } & 5.660 \\ \text { AlP } & 5.4635 \\ \text { GaAs } & 5.65325\end{array}$

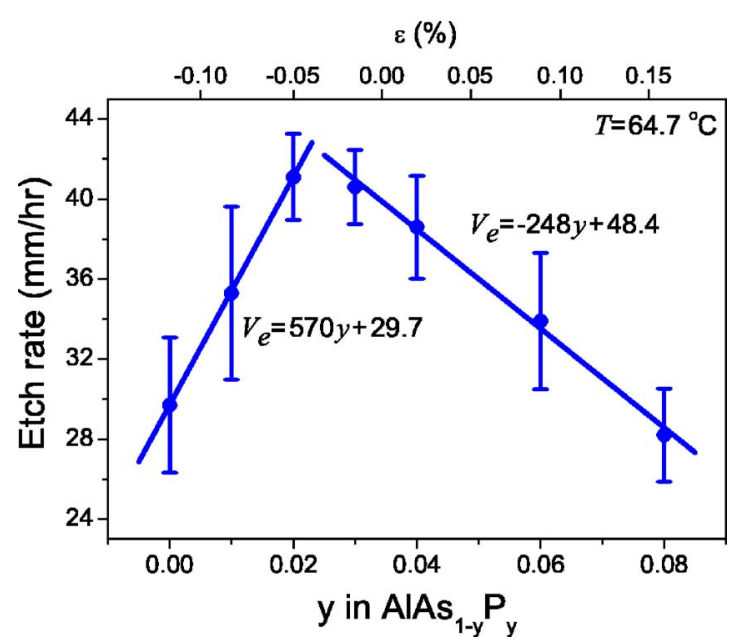

Figure 4. (Color online) Lateral etch rate in a $20 \% \mathrm{HF}$ solution as a function of the phosphorus percentage $y$ in the $10 \mathrm{~nm}$ thick $\mathrm{AlAs}_{1-y} \mathrm{P}_{y}$ layer of the structure represented by Fig. 3. The width of the error bars is given by the standard deviation, the lines are the best linear fits through the data points corresponding to a composition range varying from $y=0$ to $y=0.02$ and varying from $y=0.03$ to $y=0.08$.

lift-off of the thin-film structure from its substrate was obtained by mounting of the sample with its flexible carrier and a weight upside down on a support rod in a plastic container. Note, that the weight induced a radius of curvature $r=40 \mathrm{~mm}$ of the thin film. A 20\% HF solution was stored in a separate reservoir in which the temperature could be regulated with an accuracy of about $0.5^{\circ} \mathrm{C}$. Unless otherwise stated, this temperature was regulated in such a way that the etch temperature was $64.7^{\circ} \mathrm{C}$ for the experiments described in this work. From the reservoir, a continuous HF flow was applied to one side of the sample, and the etch time required to completely loosen the thin film from the substrate was measured. A more detailed description of the process can be found in previous work. ${ }^{13}$

After etching, the samples were rinsed in nanopure water and delicately blown dry with nitrogen. For each composition, thickness and etch temperature of the release layers 5-7 samples were processed to determine the average lateral etch rate and corresponding standard deviation. The surface morphology and roughness of the separated thin films and the substrate with buffer layer, from which they were released, were studied using a Digital Instruments Dimension 3100 atomic force microscope (AFM) in contact mode with a $\mathrm{Si}_{3} \mathrm{~N}_{4}$ tip.

\section{Results}

Etch rate.- The lateral etch rate of the ELO process as a function of the phosphorus percentage $y$ in the $10 \mathrm{~nm}$ thick $\mathrm{AlAs}_{1-y} \mathrm{P}_{y}$ release layer is shown in Fig. 4. The results reveal a maximum etch rate of $41 \pm 2 \mathrm{~mm} / \mathrm{h}$ for the $\mathrm{AlAs}_{1-y} \mathrm{P}_{y}$ samples with a $\mathrm{P}$ content of $2 \%-3 \%$. Over a composition range varying from $y=0$ to $y=0.02$ the etch rate increases for increasing P content from $30 \pm 2 \mathrm{~mm} / \mathrm{h}$ for the AlAs sample up to $41 \pm 2 \mathrm{~mm} / \mathrm{h}$ for the $\mathrm{AlAs}_{0.98} \mathrm{P}_{0.02}$ samples. The compositional change results in a reduction of the compressive strain from $-0.119 \%$ to $-0.050 \%$. A linear fit through these data points is given by

$$
V_{\mathrm{e}}=570 y+29.7
$$

with $V_{\mathrm{e}}$ the etch rate in $\mathrm{mm} / \mathrm{h}$. Over the composition range varying from $y=0.03$ to $y=0.08$ the etch rate decreases linearly for increasing $\mathrm{P}$ content from $41 \pm 2 \mathrm{~mm} / \mathrm{h}$ for the $\mathrm{AlAs}_{0.97} \mathrm{P}_{0.03}$ samples down to $28 \pm 2 \mathrm{~mm} / \mathrm{h}$ for the $\mathrm{AlAs}_{0.92} \mathrm{P}_{0.08}$ samples. This compositional variation results in a change in the intrinsic strain from a compressive strain of $-0.015 \%$ to a tensile strain of $+0.159 \%$. A linear fit through these data points can be described by 


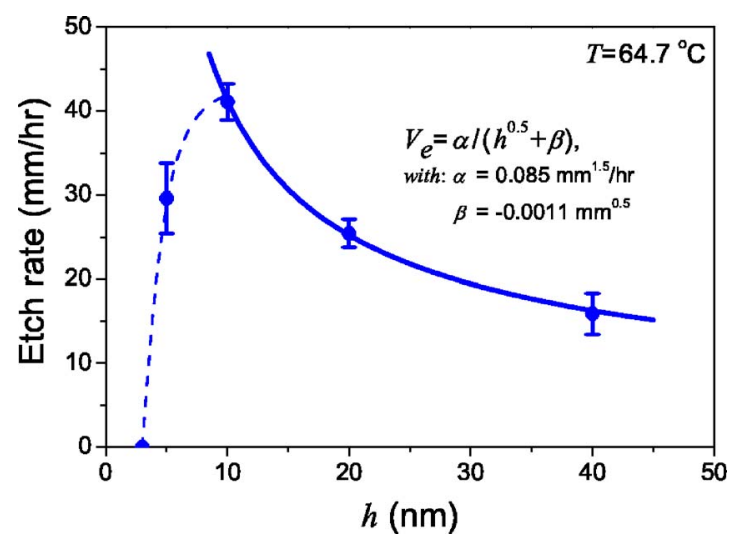

Figure 5. (Color online) Lateral etch rate of the $\mathrm{AlAs}_{0.98} \mathrm{P}_{0.02}$ release layer in a $20 \% \mathrm{HF}$ solution as a function of layer thickness $h$. The dashed line is to guide the eye and the solid line indicates the best linear fit through the data obtained for $h \geqslant 10 \mathrm{~nm}$.

$$
V_{e}=-248 y+48.4
$$

Thus, it is found that the correlation between etch rate and strain differs significantly for the two different composition regimes under the same etching conditions.

Based on these results an $\mathrm{AlAs}_{0.98} \mathrm{P}_{0.02}$ release layer was chosen as a standard for the release layer thickness series. Figure 5 shows the lateral etch rate as a function of the release layer thickness. A maximum etch rate of $41 \pm 2 \mathrm{~mm} / \mathrm{h}$ is obtained for the $10 \mathrm{~nm}$ thick $\mathrm{AlAs}_{0.98} \mathrm{P}_{0.02}$ samples, which is very similar to the optimum release layer thickness of $\sim 5 \mathrm{~nm}$ for $\mathrm{Al}_{0.85} \mathrm{Ga}_{0.15}$ As layers ${ }^{11}$ and $\sim 10 \mathrm{~nm}$ for AlAs layers. ${ }^{18}$ From Eq. 5, 9, and 12 it can be deduced that according to the DR model the relation between $V_{\mathrm{e}}$ and $h$ is described by

$$
V_{\mathrm{e}}=\frac{\alpha}{\sqrt{h}+\beta}
$$

with $\alpha$ and $\beta$ being constants. Experimentally, this relation is indeed found for samples with $h \geqslant 10 \mathrm{~nm}$ (see Fig. 5). From the best fit through the data points in this range, it can be deduced that under the present conditions $\alpha=0.085 \mathrm{~mm}^{1.5} / \mathrm{h}$ and $\beta=-0.0011 \mathrm{~mm}^{0.5}$. For $h<10 \mathrm{~nm}$, there is a clear deviation from the behavior predicted by the DR model. For samples with release layer thicknesses of 3 and $5 \mathrm{~nm}$ etch rates are obtained of $0.0 \pm 0.5$ and $30 \pm 4 \mathrm{~mm} / \mathrm{h}$, respectively. Note, that for the $3 \mathrm{~nm} \mathrm{AlAs}{ }_{0.98} \mathrm{P}_{0.02}$ samples no visible separation of the thin film and the substrate was observed after $2 \mathrm{~h}$ of etching. Therefore, the ELO process of these samples was terminated prematurely. After removing the foil and the double-sided tape, all $3 \mathrm{~nm}$ thick $\mathrm{AlAs}_{0.98} \mathrm{P}_{0.02}$ samples were carefully examined, especially the rims which were exposed to the HF flow. No traces of etch damage, like broken-out thin-film remnants, were found indicating that indeed no etching had occurred.

Corresponding to the optimum etch rate a $10 \mathrm{~nm} \mathrm{AlAs}{ }_{0.98} \mathrm{P}_{0.02}$ release layer was chosen as a standard for the experiments conducted at different temperatures the results of which are shown in Fig. 6. The average times to separate the thin film from the substrate are $61 \pm 9$ and $12.0 \pm 0.2$ min at $23.4 \pm 0.5$ and $71.2 \pm 0.5^{\circ} \mathrm{C}$, respectively. For the $10 \times 10 \mathrm{~mm}^{2}$ samples, this corresponds to minimum and maximum etch rates of $10 \pm 1$ and $47.6 \pm 0.8 \mathrm{~mm} / \mathrm{h}$ at 23.4 and $71.2^{\circ} \mathrm{C}$, respectively. From Eq. 5, 9, and 12 it can be deduced that according to the DR model the relation between $V_{\mathrm{e}}$ and $T$ is given by

$$
V_{\mathrm{e}} \sim\left[R_{\mathrm{d}, \mathrm{o}} \exp \left(E_{\mathrm{a}, \mathrm{d}} / k_{\mathrm{B}} T\right)+R_{\mathrm{r}, \mathrm{o}} \exp \left(E_{\mathrm{a}, \mathrm{r}} / k_{\mathrm{B}} T\right)\right]^{-1}
$$

with $R_{\mathrm{d}, \mathrm{o}}$ and $R_{\mathrm{r}, \mathrm{o}}$ being the diffusion rate and reaction rate resistances, respectively, at infinite temperature. For $R_{\mathrm{d}, \mathrm{o}} \exp \left(E_{\mathrm{a}, \mathrm{d}} / k_{\mathrm{B}} T\right)$ $\gg R_{\mathrm{r}, \mathrm{o}} \exp \left(E_{\mathrm{a}, \mathrm{r}} / k_{\mathrm{B}} T\right), V_{\mathrm{e}}$ is diffusion controlled and the activation

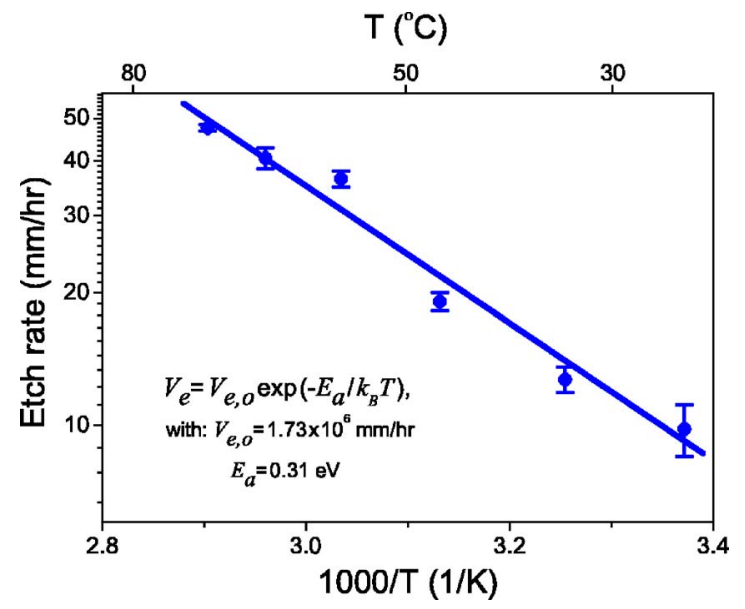

Figure 6. (Color online) Lateral etch rate of $10 \mathrm{~nm}$ thick $\mathrm{AlAs}_{0.98} \mathrm{P}_{0.02}$ release layers in a $20 \% \mathrm{HF}$ solution as a function of temperature. The solid line is the best linear fit through the data points from which an activation energy $E_{\mathrm{a}}=0.31 \mathrm{eV}$ is deduced.

energy of the overall etch rate given by $E_{\mathrm{a}, \mathrm{d}}$. For $R_{\mathrm{d}, \mathrm{o}} \exp \left(E_{\mathrm{a}, \mathrm{d}} / k_{\mathrm{B}} T\right) \ll R_{\mathrm{r}, \mathrm{o}} \exp \left(E_{\mathrm{a}, \mathrm{r}} / k_{\mathrm{B}} T\right), V_{\mathrm{e}}$ is reaction controlled and the activation energy of the overall etch rate given by $E_{\mathrm{a}, \mathrm{r}}$. If the diffusion rate resistance is of the same order of magnitude as the reaction rate resistance, then the difference in temperature dependence of the two processes can be used to alter the activation energy of the overall etch rate for the ELO process. ${ }^{19}$ This transition from the diffusion controlled regime (at low temperatures) toward the reaction controlled regime (at high temperatures) is continuous. Measurements of the activation energy in the transition regime will, therefore, lead to an activation energy that lies between the activation energies $E_{\mathrm{a}, \mathrm{d}}$ and $E_{\mathrm{a}, \mathrm{r}}$. As shown in Fig. 6, the experimentally obtained data can be described by

$$
V_{\mathrm{e}}=V_{\mathrm{e}, \mathrm{o}} \exp \left(\frac{-E_{\mathrm{a}}}{k_{\mathrm{B}} T}\right)
$$

with $V_{\mathrm{e}, \mathrm{o}}$ the etch rate at infinite temperature, and $E_{\mathrm{a}}$ the activation energy of the overall process. The best fit of this relation through the data points indicates that under the present conditions $V_{e, o}$ $=1.73 \cdot 10^{6} \mathrm{~mm} / \mathrm{h}$ and $E_{\mathrm{a}}=0.31 \mathrm{eV}$.

Surface morphology and roughness.- After separation the newly exposed surfaces at both sides of the release layer (i.e., the surfaces of the thin film and of the substrate with buffer layer from which they were released) were studied in detail using atomic force microscopy (AFM). The surface morphology of the thin film and substrate sides appeared highly similar. However, because their flexible carriers generally were slightly curved it was more difficult to obtain a high resolution AFM image from the thin-film side of the samples. Therefore, all images shown in the present work were obtained from the easy to handle substrate side of the samples.

The surfaces resulting from the $10 \mathrm{~nm}$ thick $\mathrm{AlAs}_{1-y} \mathrm{P}_{y}$ samples with various phosphorus compositions are shown in Fig. 7. Surfaces obtained from samples with AlAs release layers $(y=0)$ reveal irregularly shaped deposits with typical lateral dimensions of $0.05-0.10 \mu \mathrm{m}$ covering the entire surface (see Fig. 7a). For the $\mathrm{AlAs}_{0.98} \mathrm{P}_{0.02}$ samples (see Fig. $7 \mathrm{~b}$ ), these deposits have become larger, typically $0.1-0.3 \mu \mathrm{m}$ in lateral size. The $\mathrm{AlAs}_{0.96} \mathrm{P}_{0.04}$ and $\mathrm{AlAs}_{0.94} \mathrm{P}_{0.06}$ samples yield similar surfaces revealing deposits of $0.1-0.2 \mu \mathrm{m}$ in size with more rounded boundaries as shown in Fig. 7c. This figure also shows that for these samples the surfaces are only partly $(\sim 70 \%)$ covered; the rather smooth areas in between are probably the GaAs surface. For the $\mathrm{AlAs}_{0.92} \mathrm{P}_{0.08}$ samples, the typical dimensions of the deposits have abruptly increased to $0.5-1.0 \mu \mathrm{m}$. Figure $7 \mathrm{~d}$ shows that on the entire surface of these 
a)

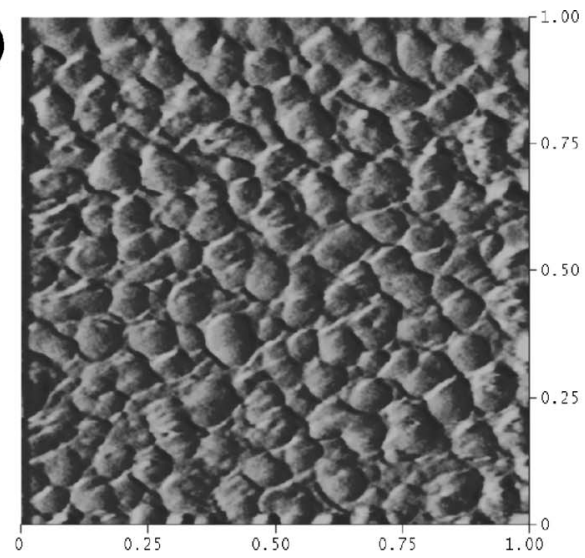

b)

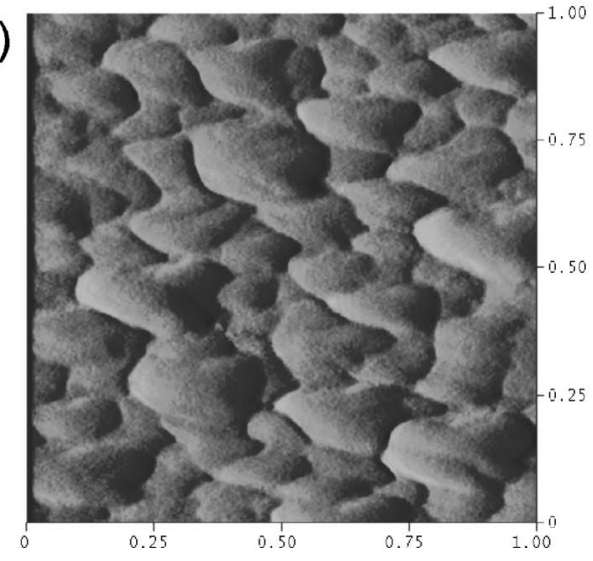

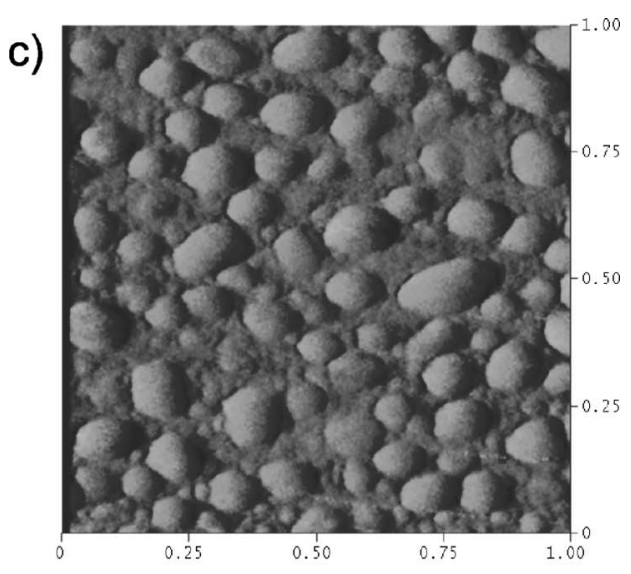

d)

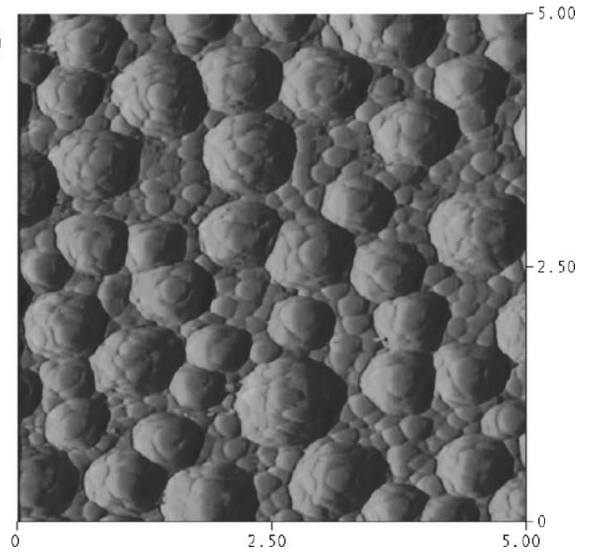

Figure 7. AFM images of the substrate surface sides of the $10 \mathrm{~nm}$ thick $\mathrm{AlAs}_{1-y} \mathrm{P}_{y}$ samples with phosphorus percentage of (a) $0 \%$, (b) $2 \%$, (c) $4 \%$ and (d) $8 \%$. The scale of the images is given in micrometers. Note that the scale of (d) is different from the others. samples a substructure of slowly sloping deposits $(0.2-0.4 \mu \mathrm{m}$ in size) has formed. AFM examination of the surface morphology was also conducted for the samples of the thickness series. For the $5 \mathrm{~nm}$ samples, slowly sloping deposits are found of $0.2-0.4 \mu \mathrm{m}$ in lateral size covering the entire surface area (see Fig. 8a). As already shown in Fig. 7b, deposits of typically $0.1-0.3 \mu \mathrm{m}$ in size are encountered on the $10 \mathrm{~nm}$ samples. For the $20 \mathrm{~nm}$ samples, a similar morphology is obtained as for the 5 and $10 \mathrm{~nm}$ samples (see Fig. 8b). For the $40 \mathrm{~nm}$ sample, however, the morphology changed: surfaces are only partly covered with slowly sloping deposits having typical dimensions of $0.3-0.5 \mu \mathrm{m}$ (see Fig. 8c). Between the deposits a rather smooth surface is found with locally some deposits of $\sim 0.1 \mu \mathrm{m}$ in size. For all samples of the temperature series, a surface morphology is found with sloping deposits of $0.1-0.4 \mu \mathrm{m}$ in lateral size, which is very similar to the morphology of the $\mathrm{AlAs}_{0.98} \mathrm{P}_{0.02}$ and $\mathrm{AlAs}_{0.96} \mathrm{P}_{0.04}$ samples (see Fig. $7 \mathrm{~b}$ and $\mathrm{c}$ ).

For the $\mathrm{AlAs}_{1-y} \mathrm{P}_{y}$ samples over a composition range from $y$ $=0$ to $y=0.06$ the root-mean-square $(\mathrm{rms})$ roughness as deduced for $1 \times 1 \mu \mathrm{m}^{2}$ AFM scans varies between approximately 2 and $6 \mathrm{~nm}$. For the $\mathrm{AlAs}_{0.92} \mathrm{P}_{0.08}$ samples, however, the rms values show an abrupt increase to $29 \pm 5 \mathrm{~nm}$ for surfaces covered with the large scale deposits with substructure on top and between these deposits. Detailed measurements of the substructure, between the large scale deposits, revealed a surface roughness of $12 \pm 4 \mathrm{~nm}$. The $\mathrm{AlAs}_{0.98} \mathrm{P}_{0.02}$ samples with various release layer thicknesses show for the 10 and $20 \mathrm{~nm}$ samples roughness values between approximately 2 and $6 \mathrm{~nm}$. For the 5 and $40 \mathrm{~nm}$ samples the highest rms values are found of $7 \pm 3$ and $9 \pm 3 \mathrm{~nm}$, respectively. For the $10 \mathrm{~nm}$ $\mathrm{AlAs}_{0.98} \mathrm{P}_{0.02}$ samples exposed to temperatures varying from 23.4 to $71.2^{\circ} \mathrm{C}$, the surface roughness varies approximately between 2 and $7 \mathrm{~nm}$, with the highest rms values measured for the 23.4 and $71.2^{\circ} \mathrm{C}$ samples.

Experimentally, it is found that the deposits on all samples could be wiped away by firm polishing with a cotton bud. After this pro- cedure, the samples reveal relatively flat surfaces with a rms value $<1 \mathrm{~nm}$ (see Fig. 9). This roughness is similar to that of the GaAs wafer before growth. ${ }^{13}$ In previous work, ${ }^{15}$ it was shown that $\mathrm{AlF}_{3} \cdot 3 \mathrm{H}_{2} \mathrm{O}$ is the only solid reaction product of the AlAs reaction with HF (see Eq. 3). Therefore, it is likely that this compound is deposited on the GaAs surfaces during the ELO process.

\section{Discussion}

Qualitative correlation of experimental findings with the $D R$ model.- In the present work, it is found that the lateral etch rate of the $\mathrm{AlAs}_{1-y} \mathrm{P}_{y}$ samples is highest for a $\mathrm{P}$ fraction of $2-3 \%$. At this composition the $\mathrm{AlAs}_{1-y} \mathrm{P}_{y}$ layer is almost lattice matched with the surrounding GaAs layers, thus it can be concluded that the maximum etch rate is obtained for an almost unstrained release layer. This correlates well with the fact that in previous work ${ }^{13}$ it was observed that the ELO etch rate maximizes for layer structures with the least amount of strain in the two $\mathrm{In}_{x} \mathrm{Ga}_{1-x}$ As or two $\mathrm{GaAs}_{1-y} \mathrm{P}_{y}$ layers surrounding the AlAs release layer. This dependence of the lateral etch rate on the strain in the release layer structure is a strong indication that under these conditions the ELO process is hampered by reaction rate limitations. Strain changes the bond lengths and angles between the bonds, thereby affecting the reaction rate constant $k$ of $\mathrm{AlAs}_{1-y} \mathrm{P}_{y}$ etching in HF.

For release layer thicknesses $\geqslant 10 \mathrm{~nm}$, a $V_{\mathrm{e}}=\alpha /(\sqrt{h}+\beta)$ relation is found between $V_{\mathrm{e}}$ and $h$, which is in accordance with the DR model presented in this article. For $h<10 \mathrm{~nm}$ the lateral etch rate of $\mathrm{AlAs}_{0.98} \mathrm{P}_{0.02}$ decreases rapidly and is zero for release layers with a thickness of $3 \mathrm{~nm}$. These findings are consistent with the results obtained in previous work. ${ }^{11}$ The data obtained for $\mathrm{Al}_{0.85} \mathrm{Ga}_{0.15} \mathrm{As}$ release layers etched at room temperature in a $10 \% \mathrm{HF}$ solution could also be fitted with a $V_{\mathrm{e}}=\alpha /(\sqrt{h}+\beta)$ relation yielding $\alpha$ $=0.0025 \mathrm{~mm}^{1.5} / \mathrm{h}$ and $\beta=-0.0012 \mathrm{~mm}^{0.5}$. In that situation, the relation was found to hold down to a thickness of $5 \mathrm{~nm}$ before the etch 


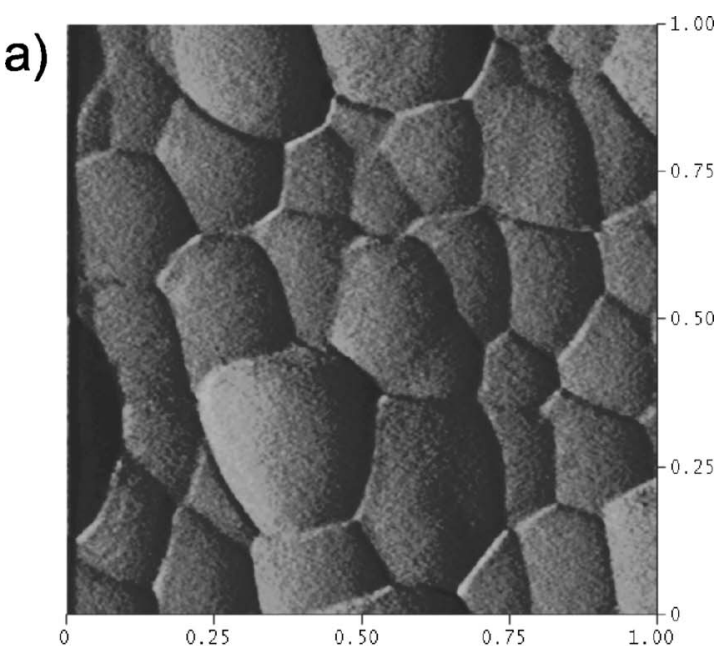

b)

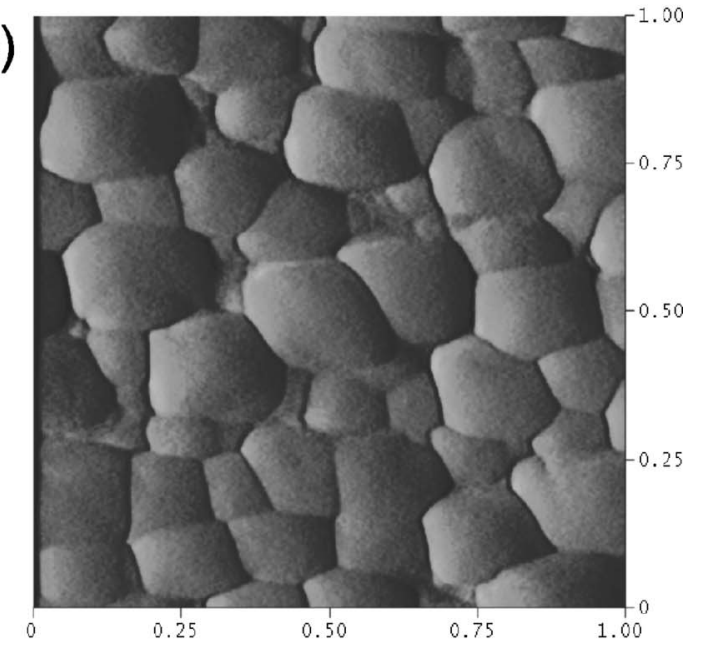

c)

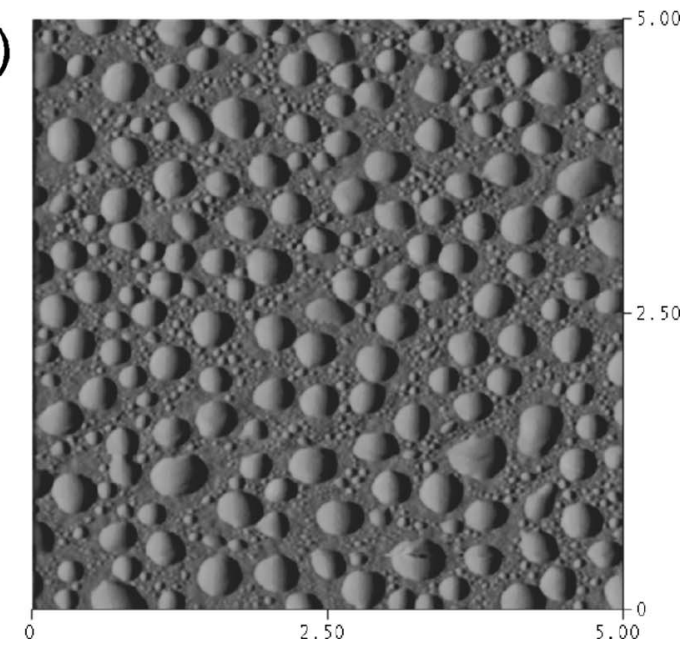

Figure 8. A typical AFM image of the substrate surface sides of the $\mathrm{AlAs}_{0.98} \mathrm{P}_{0.02}$ samples with a layer thickness of (a) $5 \mathrm{~nm}$, (b) $20 \mathrm{~nm}$ and (c) $40 \mathrm{~nm}$. The scale of the images is given in micrometers. Note that the scale of (c) is different from the others.

rate decreased and became zero at $2 \mathrm{~nm}$. This clearly indicates that for small values of $h$, the etch process is blocked in some way, such as via the formation of deposits (probably solid $\mathrm{AlF}_{3} \cdot 3 \mathrm{H}_{2} \mathrm{O}$ ) close to the etch front.

As schematically illustrated in Fig. 10, the deposition of reaction product on the thin film and substrate surfaces decreases the height

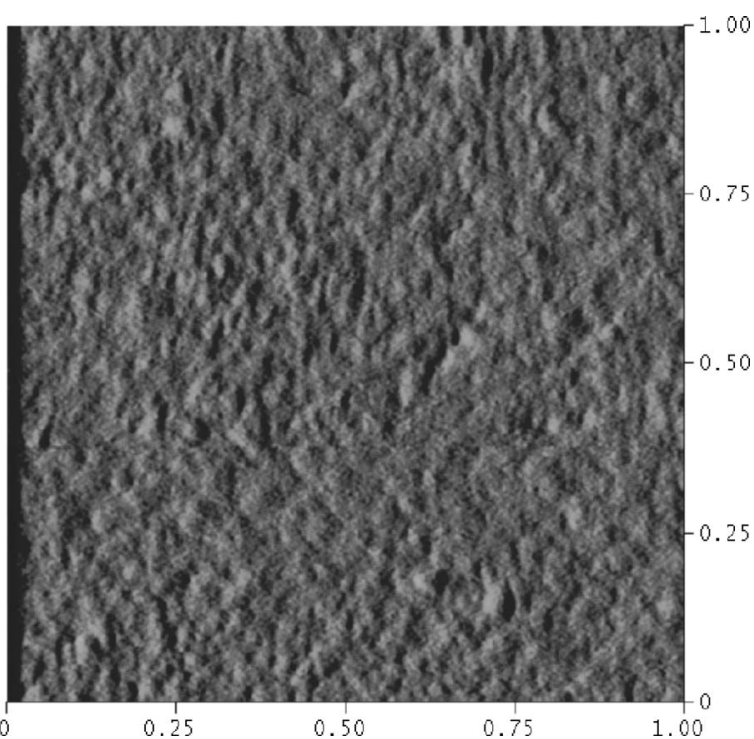

Figure 9. A typical AFM image of the substrate surface sides of the $\mathrm{AlAs}_{1-y} \mathrm{P}_{y}$ samples after the cleaning procedure. The scale of the image is given in micrometers.

$h$ of the etch slit to an effective height $h_{\text {eff }}<h$, thereby changing the diffusion resistance (see Eq. 5). For the $5 \mathrm{~nm}$ samples, a surface roughness of $\sim 7 \mathrm{~nm}$ is observed on the thin film as well as the substrate surfaces. This deposited layer might locally fill the etch slit and thereby limit the ELO process. For the $3 \mathrm{~nm}$ samples, no etching was observed, indicating that the deposition of reaction product completely blocks the ELO process. The narrowing of the etch slit by the deposition of reaction products might also explain why in both the present and a previous study ${ }^{11}$ negative values were found for constant $\beta$ in Eq. 16, while based on Eq. 5 a positive value is expected. In previous work, it was argued that the negative value could be related to evaporation of the single droplet of HF used at that time. However, in the present study evaporation of the HF droplet is excluded as a possible cause by use of a modified setup that allows for a continuous flow of HF solution towards the etch front.

Quantitative correlation of experimental findings with the $D R$ model.- Earlier ELO investigations performed by Schermer et al. ${ }^{11}$ showed that the ELO process is associated with an activation energy $E_{a}$ of $0.22 \mathrm{eV}$ for AlAs release layers etched by a single $10 \% \mathrm{HF}$ droplet, compared to $E_{\mathrm{a}}=0.31 \mathrm{eV}$ obtained in the present work for $\mathrm{AlAs}_{0.98} \mathrm{P}_{0.02}$ release layers etched by a continuous $20 \% \mathrm{HF}$ flow. This difference in activation energy is consistent with a situation in which the rate limitation of the ELO process shifts from diffusion of $\mathrm{HF}$ to the chemical reaction at the release layer surface. For $\mathrm{In}_{0.5} \mathrm{Ga}_{0.5} \mathrm{P}$ etching with a $\mathrm{HCl}$ solution a similar change in activation energy was reported in the literature. ${ }^{20}$ At a $4 \mathrm{M} \mathrm{HCl}$ solution,

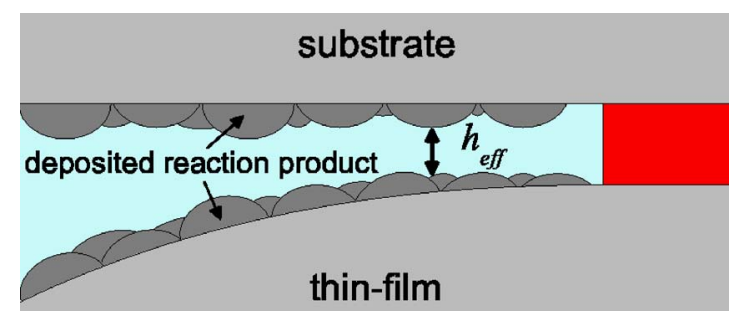

Figure 10. (Color online) Schematic representation of the situation after deposition of reaction product (probably solid $\mathrm{AlF}_{3} \cdot 3 \mathrm{H}_{2} \mathrm{O}$ ) on the thin-film and substrate surfaces during the ELO process. The height $h$ of the etch slit, as shown in Fig. 1, decreases to an effective height $h_{\text {eff }}<h$. 
the $\operatorname{~n}_{0.5} \mathrm{Ga}_{0.5} \mathrm{P}$ etch process appeared to be limited by the diffusion of $\mathrm{HCl}$ (with $E_{\mathrm{a}}=\sim 0.11 \mathrm{eV}$ ), while for increasing concentrations a transition takes place to the reaction-limited regime, yielding $E_{\text {a }}$ $=\sim 0.38 \mathrm{eV}$ for a $12 \mathrm{M} \mathrm{HCl}$ solution.

The question remains how to extract $E_{\mathrm{a}, \mathrm{d}}$ and $E_{\mathrm{a}, \mathrm{r}}$ from $E_{\mathrm{a}}$ (see Eq. 17 and 18). Since virtually nothing is known about the reaction kinetics of AlAs with $\mathrm{HF},{ }^{15}$ information is required on the diffusion data ( $D$ and $\left.E_{\mathrm{a}, \mathrm{d}}\right)$ for HF in HF solutions. Unfortunately, we were also unable to trace these data in the literature. However, we could find data related to the self-diffusion of HF (i.e., diffusion of HF in HF) from Ref. 21. So for the time being, we will use these data $\left(D=5.51 \times 10^{-5} \mathrm{~cm}^{2} / \mathrm{s}\right.$ at $283.7 \mathrm{~K}$ and $\left.E_{\mathrm{a}, \mathrm{d}}=0.10 \mathrm{eV}\right)$ as a firstorder approximation to describe the diffusion of $\mathrm{HF}$ in a $20 \% \mathrm{HF}$ solution.

In the present work, the ELO process is typically conducted with $r=40 \mathrm{~mm}, h=10 \mathrm{~nm}$ (except for the thickness series) and $T$ $=64.7^{\circ} \mathrm{C}$ (except for the temperature series). Substituting these values in Eq. 4 and 5 results in

$$
V_{\mathrm{e}, \mathrm{d}}=7.94 \cdot\left([\mathrm{HF}]_{\ell=L}-[\mathrm{HF}]_{\ell=0}\right)
$$

with $V_{\mathrm{e}, \mathrm{d}}$ the etch rate in $\mathrm{mm} / \mathrm{h}$ and the HF concentrations $[\mathrm{HF}]_{\ell=L}$ and $[\mathrm{HF}]_{\ell=0}$ in M. With a density of $\sim 1.00 \mathrm{~g} / \mathrm{cm}^{3}$ and a molar mass of $20.00 \mathrm{~g} / \mathrm{mol},{ }^{10}[\mathrm{HF}]_{\ell=L}$ is calculated to be $10 \mathrm{M}$. For the $\mathrm{AlAs}_{1-y} \mathrm{P}_{y}$ series with etch rates between 28 and $41 \mathrm{~mm} / \mathrm{h},[\mathrm{HF}]_{\ell=0}$ values are deduced between 6.4 and $4.8 \mathrm{M}$, respectively. Since the diffusion of $\mathrm{HF}$ and its reaction with the release layers are connected in series, under these conditions Eq. 12 becomes for the $\mathrm{AlAs}_{0.98} \mathrm{P}_{0.02}$ layers

$$
V_{\mathrm{e}, \mathrm{r}}=8.5 \cdot[\mathrm{HF}]_{\ell=0}
$$

Using this equation for the thickness series with etch rates between 16 and $41 \mathrm{~mm} / \mathrm{h}$ (for $h \geqslant 10 \mathrm{~nm}$ ), we obtain $[\mathrm{HF}]_{\ell=0}$ values between 1.9 and $4.8 \mathrm{M}$, respectively. For the temperature series, with etch rates between 10 and $47.6 \mathrm{~mm} / \mathrm{h},[\mathrm{HF}]_{\ell=0}$ values between 8.1 and 4.2 M respectively, are obtained. These results indicate that the DR model gives realistic $[\mathrm{HF}]_{\ell=0}$ values for all conditions $(h$ $\geqslant 10 \mathrm{~nm}$ ) applied in the present work, i.e., the concentration difference for the diffusion rate as well as for the reaction rate lies between 0 and $[\mathrm{HF}]_{\ell=L}$. By fitting Eq. 5, 9, and 12 to Eq. 18, the values $A=1.5 \cdot 10^{-10} \mathrm{~m}^{4} / \mathrm{mol} \mathrm{s}$ and $E_{\mathrm{a}, \mathrm{r}}=0.43 \mathrm{eV}$ are obtained. Unfortunately, we were unable to derive comparable data for the Arrhenius constant $A$. However, we could find data related to the activation energy for kinetically controlled reactions, which is in the order of $\sim 0.4 \mathrm{eV} .^{20,22}$ This shows that the first-order approximation to describe the diffusion of HF in HF solutions by the self-diffusion of HF gives realistic values for the activation energy of the reactionlimited process.

\section{Conclusion}

In the present work a DR model is derived based on the notion that the overall etch rate in the ELO process is determined both by the diffusion rate of $\mathrm{HF}$ to the etch front and its subsequent reaction rate in the process. In contrast to the model that was previously described in the literature, the DR model yields etch rates which are in quantitative agreement with those obtained experimentally. In order to verify the DR model, the ELO etch rate $V_{\mathrm{e}}$ of $\mathrm{AlAs}_{1-y} \mathrm{P}_{y}$ release layers is determined as a function of the phosphorus percentage (and corresponding strain), the release layer thickness and the temperature. For the AlAs ${ }_{1-y} \mathrm{P}_{y}$ series, it is found that $V_{e}$ is maximum for a $\mathrm{P}$ content of $2 \%-3 \%$, which corresponds to an almost unstrained release layer. Furthermore, it is shown that the etch rate decreases significantly for increasing compressive and tensile strain in the release layer, clearly indicating that the ELO process involves a reaction rate related component. For the thickness series, a maxi- mum etch rate is obtained for $h=10 \mathrm{~nm}$, while in accordance with the DR model a $V_{\mathrm{e}}=\alpha /(\sqrt{h}+\beta)$ relation is observed $h \geqslant 10 \mathrm{~nm}$. For the temperature series, the minimum and maximum etch rates found are $10 \pm 1$ and $47.6 \pm 0.8 \mathrm{~mm} / \mathrm{h}$ at 23.4 and $71.2^{\circ} \mathrm{C}$, respectively. From this series, an overall activation energy $E_{\mathrm{a}}$ of $0.31 \mathrm{eV}$ is deduced for the ELO process. Using the diffusion coefficient $(D$ $=5.51 \times 10^{-5} \mathrm{~cm}^{2} / \mathrm{s}$ at $\left.283.7 \mathrm{~K}\right)$ and the activation energy $\left(E_{\mathrm{a}, \mathrm{d}}\right.$ $=0.10 \mathrm{eV}$ ) for the self-diffusion of $\mathrm{HF}$ as a first-order approximation for the diffusion of $\mathrm{HF}$ in a $20 \% \mathrm{HF}$ solution an activation energy of $0.43 \mathrm{eV}$ and an Arrhenius constant of $A=1.5$ $\times 10^{-10} \mathrm{~m}^{4} / \mathrm{mol} \mathrm{s}$ could be deduced for the reaction of $\mathrm{AlAs}_{0.98} \mathrm{P}_{0.02}$ with HF.

AFM measurements revealed deposits (probably solid $\mathrm{AlF}_{3} \cdot 3 \mathrm{H}_{2} \mathrm{O}$ ) from the etch process on the surfaces at both sides of the etch slit. It is found that these deposits form continuous layers with a surface roughness of several nanometers. For release layers with a thickness $<10 \mathrm{~nm}$, these deposits start to have a large influence on the etch process, resulting in a rapid decrease of the etch rate from a maximum of $41 \pm 2 \mathrm{~mm} / \mathrm{h}$ at $10 \mathrm{~nm}$ to $0 \mathrm{~mm} / \mathrm{h}$ at $3 \mathrm{~nm}$. The occurrence of this mechanism for $h<10 \mathrm{~nm}$ was also observed in several former studies and is not yet accounted for in the DR model.

\section{Acknowledgments}

The authors thank W. H. M. Corbeek for his technical support regarding the construction of the WIELO setup and the Dutch Technology Foundation STW for financial support under project number NET.7452.

Radboud University Nijmegen assisted in meeting the publication costs of this article.

\section{References}

1. G. J. Bauhuis, J. J. Schermer, P. Mulder, M. M. A. J. Voncken, and P. K. Larsen, Sol. Energy Mater. Sol. Cells, 83, 81 (2004).

2. A. van Geelen, P. R. Hageman, G. J. Bauhuis, P. C. van Rijsingen, P. Schmidt, and L. J. Giling, Mater. Sci. Eng., B, 45, 162 (1997).

3. Y. Yazawa, J. Minemura, K. Tamura, S. Watahiki, T. Kitatam, and T. Warabisako, Sol. Energy Mater. Sol. Cells, 50, 163 (1998).

4. J. Maeda, Y. Sasaki, N. Dietz, K. Shibahara, S. Yokoyama, S. Miyazaki, and M Hirose, Jpn. J. Appl. Phys., Part 1, 36, 1554 (1997).

5. Y. Sasaki, T. Katayama, T. Koishi, K. Shibahara, S. Yokoyama, S. Miyazaki, and M. Hirose, J. Electrochem. Soc., 146, 710 (1999).

6. G. J. Bauhuis, P. Mulder, J. J. Schermer, E. J. Haverkamp, J. van Deelen, and P. K. Larsen, in Proceedings of the 20th European Photovoltaic Solar Energy Confer ence, Barcelona, Spain, p. 468 (2005).

7. M. A. Green, K. Emery, D. L. King, S. Igari, and W. Warta, Prog. Photovoltaics, 13, 387 (2005)

8. M. Konagai, M. Sugimoto, and K. Takahashi, J. Cryst. Growth, 45, 277 (1978).

9. E. Yablonovitch, T. Gmitter, J. P. Harbison, and R. Bhat, Appl. Phys. Lett., 51, 2222 (1987).

10. D. R. Lide, Editor, CRC Handbook of Chemistry and Physics, Internet Version 2007, 87th edition, http://www.hbcpnetbase. com

11. J. J. Schermer, G. J. Bauhuis, P. Mulder, W. J. Meulemeesters, E. Haverkamp, M. M. A. J. Voncken, and P. K. Larsen, Appl. Phys. Lett., 76, 2131 (2000).

12. M. M. A. J. Voncken, J. J. Schermer, G. Maduro, G. J. Bauhuis, P. Mulder, and P. K. Larsen, Mater. Sci. Eng., B, 95, 242 (2002).

13. A. T. J. van Niftrik, J. J. Schermer, G. J. Bauhuis, J. van Deelen, P. Mulder, and P. K. Larsen, Cryst. Growth Des., Accepted.

14. M. M. A. J. Voncken, J. J. Schermer, G. J. Bauhuis, A. T. J. van Niftrik, and P. K. Larsen, J. Phys.: Condens. Matter, 16, 3585 (2004).

15. M. M. A. J. Voncken, J. J. Schermer, A. T. J. van Niftrik, G. J. Bauhuis, P. Mulder, P. K. Larsen, T. P. J. Peters, B. D. de Bruin, A. Klaassen, and J. J. Kelly, J. Electrochem. Soc., 151, G347 (2004).

16. G. P. Haight, Jr., Anal. Chem., 26, 593 (1954).

17. S. M. Sze, Modern Semiconductor Device Physics, p.537, Wiley, New York (1998)

18. K. S. R. Koteswara Rao, T. Katayama, S. Yokoyama, and M. Hirose, Jpn. J. Appl. Phys., Part 2, 39, L457 (2000)

19. P. H. L. Notten, J. E. A. M. van den Meerakker, and J. J. Kelly, Etching of III-V Semiconductors: An Electrochemical Approach, Elsevier, Oxford (1990).

20. J. W. Lee, S. J. Pearton, C. R. Abernathy, W. S. Hobson, F. Ren, and C. S. Wu, Solid-State Electron., 38, 1871 (1995).

21. N. Karger, T. Vardag, and H.-D. Ludemann, J. Chem. Phys., 100, 8271 (1994).

22. Y. Mori and N. Watanabe, J. Electrochem. Soc., 125, 1510 (1978). 